\title{
Energy harvesting: a review of the interplay between structure and mechanism
}

\author{
David L. Andrews \\ Nanostructures and Photomolecular Systems, School of Chemical Sciences, \\ University of East Anglia, Norwich, NR4 7TJ, U.K. \\ david.andrews@physics.org
}

\begin{abstract}
The science of energy harvesting has recently undergone radical change, with the advent of new materials exploiting mechanisms fundamentally different from those of traditional solar cells. Utilizing principles that are in many cases acquired from breakthroughs in molecular photobiology, the introduction of a range of new synthetic polymers, multichromophore arrays and nanoparticle-based materials heralds a marked resurgence of interest, a shift of focus and heightened expectations in the science of light-harvesting. The interplay between structure and mechanism significantly impinges upon issues extending from fundamental theory to the principles of energy-harvesting materials design. Understanding and exploiting the principles allows materials to be engineered that can harness absorbed energy with heightened efficiency. Two of the key areas of application are dendrimers and rare-earth doped solids.
\end{abstract}

Keywords: Energy harvesting, solar energy, resonance energy transfer (RET), quantum electrodynamics, dendrimers, lanthanides

\section{INTRODUCTION}

The hunger for energy is a hallmark of modern civilization. Transport and industry, lighting and communications: all demand but too often squander energy, and the resource has become increasingly precious. Strenuous efforts are now being made to improve efficiency in harvesting the energy available from natural resources, especially those regarded as renewable (the term being patently ridiculous in the case of sunlight, but nonetheless entrenched): principal amongst these is solar power. For the magnitude of the resource it represents, and for its geographic availability, solar power easily outstrips any competition. Annually, the Sun delivers our planet $10^{24}$ Joules of raw energy, considerably in excess of a global demand that is not expected to reach $10^{21} \mathrm{~J}$ until the 2020's [1]. Currently, the Earth's annual energy consumption is matched by the solar energy that our planet receives in a single hour [2]. For comparison wind energy - the next largest source of environmentally available power - is a resource smaller in magnitude by a factor of more than one hundred.

There are many well-established approaches to addressing the energy problem. The best known, and widely implemented, are a variety of well-established photovoltaic schemes. These are mostly built on the concept of directly converting solar input to electrical power. Standard silicon-based photovoltaic cells routinely achieve conversion efficiencies of about $15 \%$; conversion efficiencies of up to $40 \%$ are achievable with multi-junction thin films optically tailored to span the visible spectrum [3]. Hybrid solar cells, based on inorganic nano-rods interfacing semiconducting polymers, appear to hold significant promise for generally improving solar capture efficiency [4]. Broadly similar levels of efficiency are also attainable in schemes based on the conversion of thermal energy, resulting from the absorption of solar power, into electrical form; such methods are often referred to as 'concentrating solar power' [5]. Some methods for the capture of solar energy are configured to achieve the chemical storage of energy - for example by photoelectrolysis, with the use of 
metal-ligand complexes to lower the threshold energy for decomposition of water. A longstanding aim is to provide means to support a hydrogen economy, with all its attendant advantages over fossil fuels. Hydrogen has three times the energy content of the equivalent mass of hydrocarbons, and its combustion produces no toxic by-products. However, photovoltaic cells remain too expensive to make large-scale solar electrolysis of water feasible.

Natural photosynthesis is a process whose emulation is an obvious target in energy harvesting [6]. The term 'harvesting' is indeed apposite - it signifies an integrated system for gathering natural resources, with centralized collection and distribution according to requirements. The agricultural allusion is perhaps not just incidental; many designs under evaluation exploit principles discovered in the natural photosynthetic processes that harness solar energy in chemical form. Visible light must be harvested for sustainable efficiency. However, simple consideration of the quantum-molecular energetics suggests that the mechanisms involved must be complex - also necessitating the collective action of two or more photons in some form of energy pooling. Indeed, although it has been known for over 130 years that chlorophyll mediates photosynthesis [7], the dawning of quantum theory made it evident that no single photon of visible light could supply the energy necessary to effect a breakage of chemical bonds in the carbon dioxide and water feedstock. It is now known that for each simple sugar produced, the energy of at least forty-eight visible photons is consumed; thus, the collection of photon energy is only a first step - one that has to be succeeded by pooling processes before any chemistry can begin. Ascertaining the detailed molecular structure and chromophore layout responsible for such pooling has stimulated the current renaissance in the science of solar energy capture, and many of the principles learned from photobiological systems [8] are being employed for major advances in the design of new energy-harvesting materials.

With a primary focus on the interplay between structure and mechanism, this review surveys the field. In Section 2, the key characteristics and components of an energyharvesting system are first delineated. Principal amongst these is resonance energy transfer (RET), whose fundamental electrodynamic mechanism is then discussed with reference to a simple donor-acceptor pair in Section 3. The developing understanding of this critical component has allowed the identification of various means to effect and enhance a directed character in the RET process, and the engineering of this vectorial character is the subject of Section 4. The following Section introduces the complexities that emerge in multi-site or multi-chromophore systems, and Section 6 focuses on one of the most widely studied types of biomimetic system, the fractal-stereochemistry polymers known as dendrimers. Rare-earth doped solids are addressed in Section 7, with a summing-up in the final Conclusion.

Before embarking upon this trail, a word or two of clarification is timely. First, it is interesting to observe that, as sometimes happens in science when the same or similar phrases are adopted for distinctly different subjects, the term 'energy harvesting' is also in current usage for a very different topical area. That other usage generally concerns the conversion of mechanical (usually vibrational) energy into electrical form, based on piezoelectric materials. Mostly such systems are geared more towards small-scale or portable devices, and the frequently supporting adjective 'environmental' denotes an immediate locality rather than the globe. Interested readers can find useful entries to the literature through one of several recent papers [9-13]; such subjects are not addressed in this review, which focuses specifically on the harvesting and deployment of optical radiation. Secondly, this review does not address electron transfer (its own acronym ET being one reason for the term 'resonance' commonly being added to distinguish energy transfer). The former process is of course a worthy review topic in its own right, and it is mentioned here because both in natural and many artificial photosynthetic systems, the harvesting of energy leads to ensuing electron transfer processes - marking the penultimate stage of a sequence whose completion is true chemistry. To sharpen the focus of this review, electron transfer is regarded as part of a separate tier of 
events taking place subsequent to optical energy harvesting. In certain specialized systems studied experimentally, especially those in which valence electrons are relatively delocalized, electron transfer and energy transfer can become inextricably involved with each other, leading to potentially longer-range processes such as 'superexchange' [14-17]. Again, however - to clearly demark the limits of this review and to clarify its remit - such processes are excluded from present consideration. Readers interested to explore these other matters are referred to a fine recent review by Scholes [18].

\section{LIGHT HARVESTING AT THE MOLECULAR LEVEL}

When ultraviolet or visible radiation of a suitably resonant frequency impinges on a nonhomogeneous dielectric material, the absorption of light primarily populates electronic excited states in atomic, molecular or other nanoscale constituents. A rapid degradation of the acquired energy typically ensues - largely a stochastic effect due to vibrational dissipation, with the energy ultimately to be manifest in the form of heat. Excepting cases where energy is designedly harvested through the latter's thermal action, the whole operation of an optical energy capture system is more generally based on establishing more directional and energyretaining, less random and dissipative, pathways for the flow of energy between the sites of its initial deposition, to other centers where it can be efficiently captured. To optimize harvesting efficiency, it is therefore necessary for such pathways to have a competitive edge over thermal degradation.

Several key structural and mechanistic principles can be identified as being primarily responsible for establishing preferential routes for energy flow in the energy-harvesting apparatus of biological photosystems. These same principles inform the design of many photoactive nanosystems and other synthetic light-harvesting materials; they can be listed as follows: (i) Efficient antennae are required; the chromophores responsible for initial photon absorption need broad and intense absorption bands, i.e. high value oscillator strengths (and correspondingly strong emission bands, as will emerge below). In this review, 'chromophore' will be used as a generic term for the individual particles between which energy is exchanged. In crystalline, semi-crystalline or glassy media, these centers of photon absorption (and subsequent energy release) may take the form of ions, atoms or color centers; in other types of medium they may be small molecules, electronically distinct parts of large molecules, or nanoparticles such as quantum dots; (ii) Excitation energy is generally conveyed through each system with high efficiency by resonance energy transfer, the 'resonance' signifying a loss-free character in the transfer process. For their role in each transfer event, the participating chromophores in any such medium are designated 'donor' and 'acceptor' - it being understood that any single chromophore acting in the capacity of acceptor for one transfer event may subsequently become the donor for a subsequent RET process; (iii) Following each step in a multi-step process of energy migration, losses are associated with intramolecular relaxation. Thus, as the energy progresses through a series of different chromophores, (or identical ones with increasing bathochromic shifts due to their electronic environment), a spectroscopic gradient serves to ensure directionality - a facet that is to receive detailed appraisal in Sections 3 and 4; (iv) Finally, the convergence of optically derived energy culminates in energy pooling, a process that in some systems cultivates physicochemical change, but which in other suitably tailored materials leads to optical upconversion (release of radiation with shorter wavelength); details are given in Section 7.

\section{RESONANCE ENERGY TRANSFER}

To develop a more complete understanding of these factors and their interplay, we first focus on the photophysics involved in resonance energy transfer. As is shown in the following, the propensity for energy to be transferred between any two chromophores is severely restricted by distance; if no suitable acceptor is sufficiently close, donors will generally shed their 
energy by fluorescence or local dissipation. Conversely, any flow of energy that extends beyond near-neighbors will usually comprise a series of small-scale hops between closest pairs. Pair interactions are thus not only important in systems where the absorption of optical energy leads by direct transfer to the excitation of acceptor species; such interactions are also significant as representative components of the flow in complex, multi-chromophore systems.

The primary equations for pair RET, beyond regions of wavefunction overlap, are generally derived from the formulation of an electrodynamical coupling between transition dipoles. Relatively straightforward modifications can be introduced to accommodate a degree of charge delocalization for groups in a conjugated structure [19]. The possibility of gaining calculational accuracy through adoption of a distributed monopole model [20] have to be offset against the heavy computational demands of the latter, and some loss of physical insights such as the transition dipole selection rules. It is worth observing that other forms of coupling are also possible - for example, the transfer of energy between components whose wavefunctions do significantly overlap in space is usually described in terms of Dexter theory [21], in which the coupling carries an exponential decay with distance. However, compared to materials in which the donor and acceptor orbitals lack spatial overlap, such systems are usually of less relevance for device applications. Any requirement for very small group separations undermines the efficiency of energy transfer over longer distances - such as those that separate antenna groups from the collector cores in most light-harvesting complexes. Moreover, engagement of the Dexter mechanism will usually signify a loss of individual electronic and optical integrity amongst the coupled chromophores. This is why the chromophores in complex light-harvesting systems are commonly either separated by nonconductive bonds or spacer units, or else they are embedded in a host superstructure that prevents electron exchange through 'direct' contact.

The first quantitative theory of RET was developed by Förster [22], and later experimentally verified by Latt et al. [23]. For many purposes, Förster's theory of "radiationless" energy transfer is still applicable, though from later quantum electrodynamical studies it has emerged that both "radiative" and Förster transfer are in fact the long- and shortrange limits of a more comprehensive coupling mechanism, as discussed below. To begin, consider the pairwise transfer of excitation between two chromophores, a donor $A$ and an acceptor $B$. Let it be assumed that prior excitation of the donor generates an electronically excited species $A^{*}$. Forward progress of the energy is then accompanied by donor decay to the ground electronic state. Acquiring the energy, $B$ undergoes a transition from its own ground state to an excited level. The overall RET process may thus be expressed as $A^{*}+B \rightarrow A+B^{*}$ In general, the excited acceptor, $B^{*}$, will subsequently decay in a further transfer event, or by another means such as fluorescence. Since the $A^{*}$ and $B^{*}$ excited states are real, with measurable lifetimes, the RET is fundamentally separable from prior and subsequent events, i.e. the corresponding quantum amplitude (and hence the probability) for the occurrence of this stage is factorizable from that which determines the overall sequence. Exceptions arise only in the case of multiphoton coupling processes.

\subsection{The Förster equation}

For any donor-acceptor separation, $R$, substantially smaller than the wavelengths of visible radiation - but sufficiently large for the dipole approximation to be valid - the Förster theory gives rise to the following expression for the rate of pairwise energy transfer, $w_{\mathrm{F}}$;

$$
w_{\mathrm{F}}=\frac{9 \kappa^{2} c^{4}}{8 \pi \tau_{A^{*}} n^{4} R^{6}} \int F_{A}(\omega) \sigma_{B}(\omega) \frac{d \omega}{\omega^{4}} .
$$

Here $\kappa$ is an orientation factor to be discussed below; $F_{A}(\omega)$ is the normalized fluorescence spectrum of the donor; $\tau_{\mathrm{A}^{*}}$ is the associated radiative decay lifetime; $\sigma_{B}(\omega)$ is the linear 
absorption cross-section of the acceptor, and $\omega$ is an optical frequency in radians per unit time. For systems where the donor and acceptor are accommodated within a common host material, $n$ is the refractive index at an optical frequency corresponding to the mean transferred energy [24]. Nonetheless, the linewidth of optical transitions generally manifests the influence of local electronic environments. Similarly, the presence of inhomogeneous broadening in solutions or disordered solids reflects interactions with a solvent or host, while the broad bands exhibited by chromophores in many complex molecular systems signify extensively overlapped vibrational levels - both those that are associated with intramolecular vibrations, and others associated with librations or skeletal modes of the superstructure. In each case, the broadening of electronic levels allows pair transfer to occur at any energy level encompassed by the region of overlap between the donor emission and acceptor absorption bands, as illustrated in Fig. 1 [25]. As indicated in this figure, the propensity for forward transfer is usually significantly greater than that for backward transfer, due to a sizeable difference in the spectral overlaps for the two processes: this feature, highly important in determining the efficiency of energy harvesting materials, is to be examined in more detail in Section 4. Probably the most familiar feature of Eq. (1) is its inverse sixth power dependence on $R$, often exhibited in the form $w_{\mathrm{F}} \propto\left(R_{0} / R\right)^{6}$, where $R_{0}$ (known as the Förster radius) denotes a separation at which the theoretical rates of RET and spontaneous emission by the donor become equal.

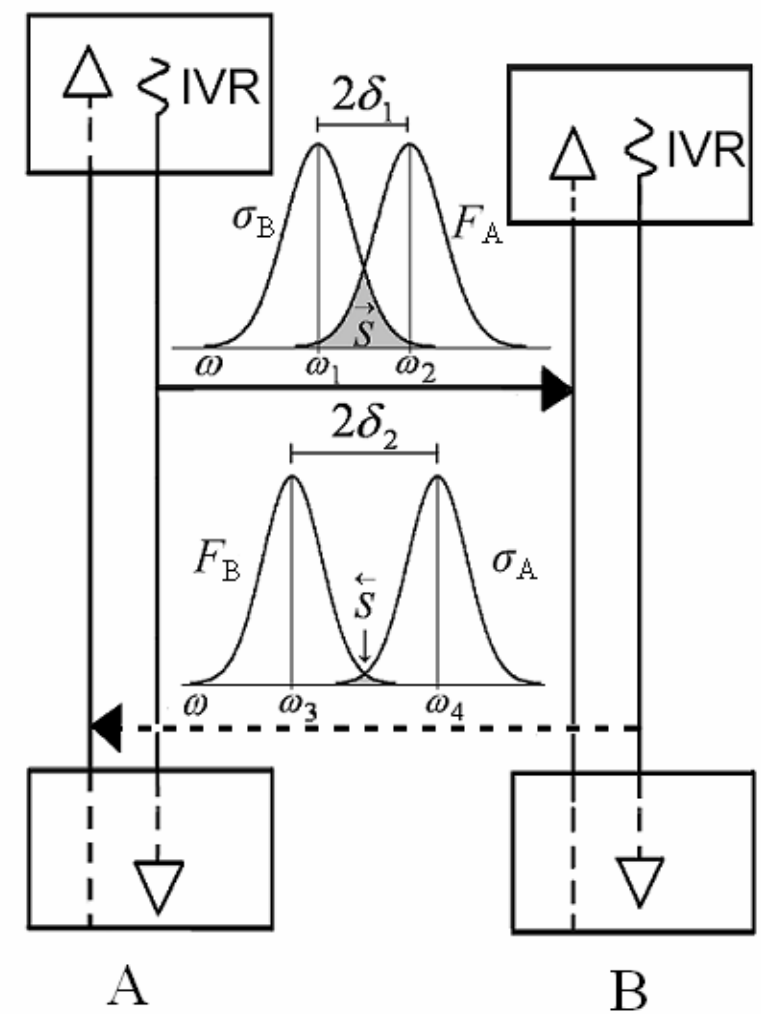

Fig. 1. Energetics for pair RET, and associated spectral overlaps: top for transfer from $A$ to $B$; below, potential backward transfer, $B$ to $A$ : $F$ - fluorescence spectrum; $\sigma$ - absorption spectrum; IVR - intramolecular vibrational (or thermal, host-mediated) dissipation. 


\subsection{Transition dipoles}

The spectral profile functions in Eq. (1) are expressible in terms of the electronic transition properties of the chromophores, taking into account their vibrational structure. Though each transition generally spans a range of frequencies within the overall spectral bandwidth, it can be assumed under the conditions of the Born-Oppenheimer approximation that the vibrational factors in the transition dipole moments factorize out as Franck-Condon factors. The spectral factors $F_{A}(\omega)$ and $\sigma_{B}(\omega)$ are then given as follows, using Dirac notation:

$$
\begin{gathered}
F_{A}(\omega)=\frac{\omega^{3} \tau_{A^{*}} \mu_{A}^{2}}{3 \varepsilon_{0} \pi c^{3}} \sum_{n, r} \rho_{A^{*}}^{(n)}\left|\left\langle\varphi_{A}^{(r)} \mid \varphi_{A^{*}}^{(n)}\right\rangle\right|^{2} \delta\left(E_{A_{n}^{*}}-E_{A_{r}}-\hbar \omega\right) ; \\
\sigma_{B}(\omega)=\frac{\pi \omega \mu_{B}^{2}}{3 \varepsilon_{0} c} \sum_{m, p} \rho_{B}^{(m)}\left|\left\langle\varphi_{B^{*}}^{(p)} \mid \varphi_{B}^{(m)}\right\rangle\right|^{2} \delta\left(E_{B_{p}^{*}}-E_{B_{m}}-\hbar \omega\right) .
\end{gathered}
$$

Here $\mu_{A}$ and $\mu_{B}$ are the magnitudes of the transition electric dipole moments for the donor decay and acceptor excitation, specifically given by:

$$
\boldsymbol{\mu}_{A}=\left\langle\psi_{A}|\boldsymbol{\mu}| \psi_{A^{*}}\right\rangle ; \boldsymbol{\mu}_{B}=\left\langle\psi_{B^{*}}|\boldsymbol{\mu}| \psi_{B}\right\rangle,
$$

where the $\boldsymbol{\mu}$ is the dipole operator and each $\psi$ is an electronic state wavefunction. The indices $m, n, p, r$ in (2) and (3) are generic labels denoting vibrational sub-levels, $\varphi$ represents an associated wavefunction and $E$ the corresponding energy; each $\rho$ denotes a population distribution function for the initial state of each species.

The $\kappa$ factor in Eq. (1), which depends on the orientations of the donor and acceptor transition dipoles, both with respect to each other, and with respect to their mutual displacement unit vector $\hat{\boldsymbol{R}}$, is defined as follows:

$$
\kappa=\left(\hat{\boldsymbol{\mu}}_{A} \cdot \hat{\boldsymbol{\mu}}_{B}\right)-3\left(\hat{\boldsymbol{R}} \cdot \hat{\boldsymbol{\mu}}_{A}\right)\left(\hat{\boldsymbol{R}} \cdot \hat{\boldsymbol{\mu}}_{B}\right) .
$$

For each chromophore, $\hat{\boldsymbol{\mu}}$ designates a unit vector in the direction of the appropriate transition dipole moment. The angular disposition of chromophores is an extremely important facet of energy transfer, inviting careful consideration in the design of lightharvesting materials. Correcting a common misconception, it is to be noted that transfer is not necessarily precluded when the transition moments lie in perpendicular directions provided that neither is orthogonal to $\boldsymbol{R}(=R \hat{\boldsymbol{R}})$. For the donor or the acceptor transition moment in molecules of sufficiently high symmetry, it can happen that either one or the other is not uniquely identifiable with a particular direction in the corresponding chromophore reference frame. Specifically, the electronic transition may then relate to a transition involving a degenerate state - as can occur with square-planar complexes, for example [26]. The considerable complication which each of these effects brings into the analysis of RET has been extensively researched and reported on by van der Meer [27].

A comparison of the above results with Eq. (1) reveals that the energy transfer rate entails a quadratic dependence on a transition dipole coupling that is algebraically isomorphic, in both its distance and orientation dependence, with the familiar interaction between electrostatic dipoles. The characteristic inverse sixth-power distance dependence of RET on 
distance thus reflects an origin in a quantum amplitude based on short-range coupling between transition electric dipoles. The result is, of course, applicable only when both the donor decay and acceptor excitation transitions are electric-dipole (E1) allowed - a point to be revisited later.

\subsection{Insights and corrections from QED}

The Förster theory is subject to several limitations. It is specifically applicable only under near-field conditions, i.e. over donor-acceptor distances significantly less than the optical wavelength for the energy being transferred; this is why the coupling has an essentially identical character to that of a static dipole interaction. (As donor-acceptor distances increase into and beyond a scale comparable with the optical wavelength for the energy being transferred, the finite timescale for causal effect lowers the threshold of energy conservation.) To ascertain rate expressions that correctly represent both short- and long-range transfer requires a fundamentally rigorous, quantum mechanical basis that delivers properly retarded solutions. The most suitable framework is afforded by quantum electrodynamics (QED) [28] whose wider successes, such as its correct predictions of the magnetic moment of the electron, the Lamb shift and the Casimir effect, are well known [29]. Less well known is the fact that even the use of electric and magnetic multipoles is ultimately defensible only in the context of a fully quantum electrodynamical theory [30]. Quite apart from the precision and rigor of the latter framework, a strong case can also be made for the value of a QED formulation on the grounds of the additional conceptual and physical insights it generates - and for the common basis it provides for understanding more complex processes such as the energy pooling to be examined in Section 5.

In connection with the theory of resonance energy transfer, the development of a theory based on QED began in pioneering work by Avery, Gomberoff and Power [31, 32], culminating in a more recent unified theory [33-36]. A suitable starting point is the following pair Hamiltonian:

$$
H=H_{A}+H_{B}+H_{\text {int }}(A)+H_{\text {int }}(B)+H_{\text {rad }} .
$$

In the above equation, the first two terms are the unperturbed Hamiltonian operators for the donor and acceptor; following them, the $H_{\text {int }}$ operators signify corresponding interactions with the radiation field, and the final term, $H_{\text {rad }}$, is the radiation Hamiltonian. Although Eq. (6) is exact, it is notable that the full Hamiltonian contains no electrostatic term directly linking $A$ with $B$. Conversely, the operator $H_{\text {rad }}$ is always involved in the system Hamiltonian (even when there are no real photons present); for this reason, any form of coupling between chromophores can only be mediated by their individual interactions with the radiation field. This is a common feature of any development in terms of multipolar transitions - it is true not only for electric dipole interactions, but for every other order of electric and magnetic multipole, even extending to the diamagnetization response [37]. On developing Eq. (6) in the electric-dipole approximation, each $H_{\text {int }}(\xi)$ system-operator is given by the usual dipole-coupling formula;

$$
H_{\text {int }}(\xi)=-\sum_{\xi} \boldsymbol{\mu}_{\xi} \cdot \boldsymbol{e}^{\perp}\left(\boldsymbol{R}_{\xi}\right)
$$

where each electric-dipole operator, $\boldsymbol{\mu}_{\xi}$, operates on matter states, and the transverse electric 
field operator, $\boldsymbol{e}^{\perp}\left(\boldsymbol{R}_{\xi}\right)$, on electromagnetic radiation states; $\boldsymbol{R}_{\xi}$ is the position vector of the chromophore labeled $\xi$. By standard methods the electric field operator is cast as a summation over optical modes, each characterized by wave-vector and polarization. Every operation of $H_{\text {int }}$ is then associated with the creation or annihilation of a photon from one of these modes.

Throughout the usual, pairwise process of RET no applied radiation is involved (recall that the initial photoexcitation and any fluorescence are physically separable events); hence the lowest order process that can couple the donor decay and the acceptor excitation transitions is one involving the creation and annihilation of a virtual photon. Such photons are not physically observed; the quantum theory accordingly requires a sum to be taken over all corresponding radiation modes (i.e. both wave-vectors and polarizations). Since $H_{\mathrm{int}}$ must feature twice - once to create the virtual photon, and the second time to annihilate it the quantum amplitude $M_{f i}$ for RET is determined by second-order perturbation theory as follows;

$$
M_{f i}=\sum_{r} \frac{\left\langle f\left|H_{\mathrm{int}}\right| r\right\rangle\left\langle r\left|H_{\mathrm{int}}\right| i\right\rangle}{\left(E_{i}-E_{r}\right)}
$$

where $i, f$ and $r$ denote initial, final and intermediate states of the system and $E$ signifies an energy. Two possible interaction sequences arise: $(a)$ the virtual photon is created at $A$ (effecting the decay of the donor excited state) and subsequently annihilated at $B$ (effecting the acceptor excitation); $(b)$ the virtual photon is created at $B$ (along with the acceptor excitation) and annihilated at $B$ (with the donor decay). These two possibilities are both represented within a state-sequence diagram as shown in Fig. 2. The counter-intuitive nature of case $(b)$ does not preclude the necessary inclusion of the corresponding quantum amplitude contribution in the calculation; it can be understood that exact energy conservation is not imposed during the interval between creation and annihilation of the virtual photon, i.e. the ultrashort photon flight-time. A key feature of virtual photon behavior, this is consistent with the time-energy uncertainty principle [38]; when the whole system enters its final state, energy conservation is once again satisfied.

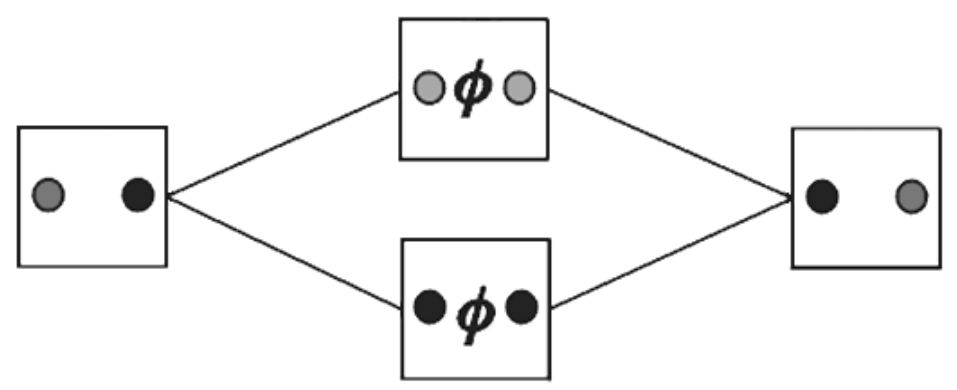

Fig. 2. State-sequence diagram, progressing from the initial system state on the left, through intermediate states, to the final state on the right. In each box two circles designate the states of $\mathrm{A}$ and $\mathrm{B}$, black indicating the ground state and grey the electronic excited state; $\phi$ denotes a virtual photon. The lower route $(a)$ and the upper route $(b)$ signify the two admissible quantum pathways for RET, consistent with time-energy uncertainty. 
Explicit evaluation of Eq. (8) by any of several standard techniques requires a considerable amount of algebra and calculus; details can be found in the original papers and recent reviews $[35,36]$. The result emerges in a form concisely expressible as;

$$
M_{f i}=\mu_{A ; i} V_{i j}(k, \boldsymbol{R}) \mu_{B ; j},
$$

where the subscript indices $i$ and $j$ stand for Cartesian components, and the convention of summation over repeated indices is implicit. The value of $k$ is $2 \pi / \lambda$, where $\lambda$ is the wavelength associated with the transfer energy. In Eq. (9), the two transition dipole moments, for the donor decay and acceptor excitation transitions, are coupled by an E1-E1 coupling tensor defined by;

$$
V_{i j}(k, \boldsymbol{R})=\frac{e^{\mathrm{i} k R}}{4 \pi \varepsilon_{0} R^{3}}\left\{\left(\delta_{i j}-3 \hat{R}_{i} \hat{R}_{j}\right)-(\mathrm{i} k R)\left(\delta_{i j}-3 \hat{R}_{i} \hat{R}_{j}\right)-(k R)^{2}\left(\delta_{i j}-\hat{R}_{i} \hat{R}_{j}\right)\right\},
$$

where the Kronecker delta tensor is defined by $\delta_{i j}=0$ if $i \neq j ; \delta_{i j}=1$ if $i=j$. The above result is the most commonly encountered instance of a more general formula [39] for the coupling between two electric multipoles $\mathrm{E} m-\mathrm{E} n$;

$$
M_{f i}=E_{A ; i_{1} \ldots i_{m}}^{(m)} V_{i_{1} \ldots i_{m} j_{1} \ldots j_{n}}(k, \boldsymbol{R}) E_{B ; j_{1} \ldots j_{n}}^{(n)},
$$

where

$$
V_{i_{1} \ldots i_{m} j_{1} \ldots j_{n}}(k, \boldsymbol{R})=\frac{(-1)^{m}}{4 \pi \varepsilon_{0}}\left(-\nabla^{2} \delta_{i_{i_{1}} j_{1}}+\nabla_{i_{1}} \nabla_{j_{1}}\right) \nabla_{i_{2}} \ldots \nabla_{i_{m}} \nabla_{j_{2}} \ldots \nabla_{j_{n}} \frac{e^{i k R}}{R}
$$

In practice, it is necessary to consider only the form of coupling effected by the lowest orders of multipole (electric or magnetic) that support the donor and acceptor transitions. In the long-range limit, every form of coupling exhibits an asymptotic dependence on $R^{-1}$, signifying a behavior that properly delivers the inverse square law for the rate of radiative energy transfer. In the present context it is more important to consider distances within the Förster radius, for which the weaker constraint $k R<<1$ invariably applies: this signifies a distance significantly smaller than the wavelength of the donor decay. (Any system in which donoracceptor closest neighbor distances failed the $k R<<1$ criterion would have the relevant species present in concentrations too low for energy-harvesting purposes.) Here, the distancedependence exhibits the limiting form $R^{-(m+n+1)}$ for the coupling of transition electric multipoles E $m$-En (or two magnetic multipoles $\mathrm{M} m-\mathrm{M} n$; for the coupling of an electric with a magnetic multipole, $\mathrm{E} m-\mathrm{M} n$, the short-range distance dependence is $R^{-(m+n)}[39,40]$.) For example, the coupling of an electric-dipole decay with an electric-quadrupole excitation, E1E2, has an $R^{-4}$ distance dependence within the Förster range. Note that each unit increase in multipolar order - and equally each substitution of an electric transition by a magnetic counterpart - lowers the strength of the coupling by a factor of the order of $10^{-2}-10^{-3}$. The decreasing efficiency of successive multipole orders increasingly disfavors the role of RET in the decay of the donor, compared to other decay mechanisms.

The QED analysis culminates in a rate law by simple application of the Fermi Rule, $w_{\mathrm{F}}=$ $2 \pi \hbar^{-1}\left|M_{f i}\right|^{2} \rho_{f}$, where $\hbar$ is the reduced Planck's constant $(h / 2 \pi)$ and $\rho_{f}$ is the density of final 
states. For the vast majority of transitions involved in energy-harvesting systems, which are electric-dipole allowed, the ensuing result in its the short-range limit reproduces Eq. (1) save that the inverse fourth power of the refractive index now appears in frequency-dependent form, within the $\omega$ integral. The feature signifies the electronic influence of other material in the vicinity of the donor and acceptor; in the case of doped solids the host crystal exerts the primary electronic influence, for example, whereas in natural photosynthetic materials it is usually the protein superstructure. Beyond the Förster regime additional terms enter the rate equation, signifying the onset of retardation effects; details of the more general behavior are given in a recent review [41]. In the primarily near-field context of energy harvesting, as will become apparent in the next and later Sections, a more significant virtue of the QED development is that it leads naturally into the conception, description and accommodation of several nonlinear effects, prominently operative in the Förster range.

\section{DESIGN PRINCIPLES FOR DIRECTED TRANSFER}

As observed earlier, a system to be used for energy harvesting should efficiently deliver electronic excitation, from sites of initial photon absorption, to centers where the energy can be pooled. A random walk would be extremely inefficient and engender an unacceptable level of dissipative losses, especially in a three-dimensional system; it is therefore important that structures and mechanisms are in place to impose a directed character upon the energy transfer stages. Although not common, one synthetically challenging but in-principle simple expedient is to employ a host structure with inbuilt channels, such as the zeolite-based materials that are being explored by Calzaferri et al. [42] Another possibility is to anchor the chromophores onto an organogel scaffold [43].

Before entertaining more general mechanisms to modify or enhance energy transfer, let us consider the factors that determine the relative propensities for the transfer of energy, in either direction, between a given chromophore pair. From the analysis in Section 3, it will be apparent that there is a close similarity of form between the equations for 'forward' and 'backward' transfer between any given pair of electronic levels. For transfer in either direction the distance considerations are clearly identical; moreover, since the unit displacement vector becomes $-\hat{\boldsymbol{R}}$ for back-transfer, its quadratic involvement in the orientation factor means that the latter, given by Eq. (5), is also the same. By reference to Eqs. (2) - (4), and considering their counterparts for the inverse transfer process, it is evident that the key to intrinsic directedness is principally a matter of differences in the donor and acceptor spectral profiles. To quantify the relative rates or propensities for forward and backward transfer it has recently been found convenient to introduce a dimensionless relative directional efficiency [25], defined by:

$$
\varepsilon=\frac{\tau_{B} \int F_{A}(\omega) \sigma_{B}(\omega) \omega^{-4} d \omega}{\tau_{A} \int F_{B}(\omega) \sigma_{A}(\omega) \omega^{-4} d \omega}
$$

For any case where the donor and acceptor are chemically different, or if these chromophores are subject to significantly different local electronic environments (either case usually manifest in identifiable differences between the corresponding absorption and emission profiles), Fig. 1 illustrates the typical implications for the spectral overlaps appearing in the numerator and denominator of (13). For each chromophore, the fluorescence peak is generally Stokes-shifted to a lower frequency with respect to its absorption counterpart. To accommodate IVR or associated losses that follow initial excitation of the donor, the acceptor will also usually have the peak of its absorption curve at a lower frequency than that of the donor emission. For such a system, forward transfer is clearly favored because (with 
reference to the parameters shown in Fig. 1) $\omega_{4}-\omega_{3}>\omega_{2}-\omega_{1}$, and $\delta_{2}>\delta_{1}$. Relatively simple results for the efficiency $\varepsilon$ emerge if the two components of the chromophore pair have similar fluorescence lifetimes, and their absorption and fluorescence curves have similar heights and full-widths at half maximum, $\omega_{\mathrm{FWHM}}$. In the specific case of Gaussian spectra, (13) then reduces to;

$$
\varepsilon \cong e^{2 k\left(\delta_{2}^{2}-\delta_{1}^{2}\right)},
$$

where $k=(4 \ln 2) \omega_{\mathrm{FWHM}}^{-2}$. The result exhibits a transfer efficiency strongly increasing with $\delta_{2}$, and decreasing with $\delta_{1}$, as might be anticipated. Furthermore, if the Stokes shifts for $A$ and $B$ are equivalent, and represented by $\omega_{S}=\omega_{4}-\omega_{2}=\omega_{1}-\omega_{3}=\delta_{2}-\delta_{1}$, and the shift characterizing the spectroscopic gradient is defined as $\omega_{G}=\omega_{4}-\omega_{1}=\omega_{2}-\omega_{3}=\delta_{1}+\delta_{2}$, the directional efficiency is expressible in its simplest form:

$$
\varepsilon=e^{2 k \omega_{G} \omega_{S}} .
$$

This result shows the same functional dependence on the spectroscopic gradient and the Stokes shift; both are equally important in determining the directedness of the energy transfer. In most energy-harvesting systems, a progressive spectroscopic gradient operating at every energy transfer step thus ensures an overall directionality of flow, a characteristic summarized in the term energy funnel. In a recent study accommodating both symmetric and asymmetric line-shapes, detailed analytical results have been determined for the directional efficiency $\mathcal{E}$ [25]. Other, experimental studies of the temperature-dependence of RET have dramatically proven the importance of molecular vibrations in determining the transfer directionality [44].

A secondary consideration is the influence of other material components, in the vicinity of a given donor-acceptor pair. It is well known that the dielectric properties of the medium within which energy is transferred (the protein matrix in photosynthetic systems, for example) exercises a considerable influence on the transfer efficiency, beyond the simple refractiveindex dependence exhibited in the Förster formula [45]. In terms of detailed mechanism, one obvious possibility in determining the efficiency of excitation transfer is mediation of the transfer through the electronic involvement of a bridge or other chemically linked species. Indeed, several studies on rigid bichromophore and multichromophore molecules have shown that in such cases a through-bond super-exchange interaction can strongly dominate intramolecular energy transport [16, 17, 46, 47]. Two other prominent mechanisms, identified through a QED analysis [48], signify more generally operative local influences of strongly polar or polarisable groups. Each type of 'third-body' chromophore, to be labeled $M_{\mu}$ and $M_{\alpha}$ respectively, has the capacity to exert its influence through a transfer process that is now chemically expressible, in either case, as $A^{*}+B+M \rightarrow A+B^{*}+M$. Consider for example a polar species $M_{\mu}$; the electric field produced by its static dipole obviously modifies the electron distributions of any neighboring chromophores. In the case where it is closest to the donor $A$, for example, the QED counterpart to Eq. (9) is:

$$
M_{f i}=\mu_{M ; k} V_{k l}\left(0, \boldsymbol{R}_{M A}\right) \alpha_{A ; i l}(-k ; 0) V_{i j}\left(k, \boldsymbol{R}_{A B}\right) \mu_{B ; j},
$$

where $\mu_{M ; k} V_{k l}\left(0, \boldsymbol{R}_{M A}\right)$ signifies the static field produced by $M_{\mu}$ and $\alpha_{A ; i}(-k ; 0)$ is an electro-optic polarizability tensor. Similarly, with a highly polarisable species $M_{\alpha}$, there is an equally significant contribution to the quantum amplitude from: 


$$
M_{f i}=\mu_{A ; i} V_{i k}\left(k, \boldsymbol{R}_{A M}\right) \alpha_{M ; k l}(-k, k) V_{l j}\left(k, \boldsymbol{R}_{A B}\right) \mu_{B ; j}
$$

in which the polarizability tensor has its usual dispersive form. The simplicity of the above results conceals not only the inverse sixth-power dependence on each relative displacement $A$ with respect to both $M$ and $B$ in (16); $M$ with respect to both $A$ and $B$ in (17) - but also a more intricate dependence on the relative orientations of the three groups - details of theoretical calculations are given elsewhere $[48,49]$. The effects are marked, a polarisable bridge readily conveying potential transfer efficiency enhancements of $50 \%-70 \%$ or more; such features clearly need to be taken seriously in the design of energy-harvesting materials. The full analysis even shows how a chemical assembly can be tailored to effectively 'switch on' the energy transfer under circumstances where the process is normally forbidden by orientational effects or on symmetry grounds.

A further factor that deserves consideration arises in the case of identical - closely neighboring but electronically distinct - donor and acceptor chromophores, where differences in behavior can arise through the possible formation of excitons. It is as well to make clear the meaning of the term exciton in the present context, since elsewhere it is employed with a wide variety of connotations - even including the single donor- or acceptor-localized excitations discussed above. In common with most recent literature on energy harvesting, however, exciton here denotes a non-propagating, delocalized electronic state associated with the quantum interference of separate chromophore states. Very simply, the excited states of any isolated pair (comprising a strongly coupled donor and acceptor) can mix and form a delocalized excited state split by twice the coupling energy. In light-harvesting complexes comprising large numbers of equivalent chromophores, excitons can spread over several equivalent donor/acceptor species and be associated with numerous, closely separated energy levels. Indeed, the separation between the lowest levels affords a useful means of gauging the extent of exciton delocalization [50]. Although any local disorder can substantially compromise the extent of such delocalization, and any superexchange coupling, one surprising finding has arisen in connection with the coupling between bacteriochlorophyll molecules. Specifically, in the case of B800-B850 energy transfer, (the numerals indicating in $\mathrm{nm}$ the wavelengths of absorption maxima), it transpires that site disorder can engender a more rapid transfer of excitation than its absence [51]. In multichromophore arrays, the multiplicity of the associated exciton splitting enhances spectral overlap and thereby accelerates energy transfer. Thus, in natural light-harvesting complexes ring structures, which provide symmetry in the pigment arrays, enhance the absorption and lead to those arrays as a whole acting as nanoscale energy traps. The mechanistic interpretations of many bacterial and other photosynthetic systems are based on exactly this principle [52-54].

\section{ENERGY POOLING IN CHROMOPHORE ARRAYS}

In natural photosynthetic systems, the delivery of individual quanta of absorbed energy to any kind of trap is insufficient to effect the mechanisms that can lock that energy in chemical form. The same principle generally applies to artificial energy harvesting materials - several quanta have to be delivered to a specific type of site for sufficient energy to be acquired and thereupon trapped. For this reason, the harvesting of energy by a multichromophore system typically employs numerous parallel channels of energy transfer directed towards the same site, where pooling of the net energy drives a subsequent conversion process. To this end, a common structural motif is a positioning of primary 'antenna' chromophores (those principally responsible for absorbing the incident radiation) on the periphery of arrays centered upon individual traps. In nature, ring structures are commonly found [52-54], whereas synthetic materials are mostly constructed on the basis of repeatedly branched dendrimers (see the next Section), phthalocyanines, or fullerenes. Indeed the first system 
proven capable of producing charge separation in direct emulation of a natural photosynthetic system was based on an array comprising five phthalocyanine molecules and a fullerene attachment [55].

In developing a detailed understanding of energy pooling, theoretical work is progressing hand in hand with current synthetic efforts. Part of the challenge lies in dealing with the complexities associated with energy transfer in systems of intricate molecular architecture $[56,57]$. With multichromophore arrays of increasing size, the complexity of the associated energy pooling calculations grows considerably, especially in connection with the selection rules and orientational factors [58]. A simplifying feature is the identification of two fundamental mechanistic motifs that act as components in any multi-chromophore, multi-step pooling process.

Consider a three-chromophore unit comprising two identical donors, $A$ and $A^{\prime}$, and one acceptor $B$ - recently reported studies on distyrylbenzene derivatives [59] afford excellent examples. The pooling process can be represented $A^{*}+A^{\prime *}+B \rightarrow A+A^{\prime}+B^{* *}$, where the double star on $B$ denotes an electronic state that is usually (though not necessarily) higher than the first excited state. Two fundamentally different mechanisms can mediate the effect, as shown in Fig. 3. In one mechanism, energy transfer involves routing from $A$ to $B$ via $A^{\prime}$, at which intermediate point the additional excitation energy of $A^{\prime}$ is acquired. The resultant pooling of energy at $B$, which should not be thought of as a two-step process since energy need not be conserved before its completion, has been designated an accretive mechanism with due regard to the nature of the intermediary role of $A^{\prime}$; the roles played by $A$ and $A^{\prime}$ are of course interchangeable. The overall process is regarded as proceeding in stepwise fashion because the donor excited states have a finite lifetime before energy transfer. A second mechanism, by means of which energy is deposited at $B$ directly from the two donors, is termed cooperative [60]. The general characteristics of both mechanisms have been determined by detailed QED appraisal [58], leading to the following quantum amplitudes:

$$
\begin{gathered}
M_{\text {coop }}=\mu_{A ; i} V_{i k}\left(k, \boldsymbol{R}_{A B}\right) \sigma_{B ; k l}(k, k) V_{l j}\left(k, \boldsymbol{R}_{A^{\prime} B}\right) \mu_{A^{\prime} ; j}, \\
M_{\text {acc }}=\mu_{A ; i} V_{i k}\left(k, \boldsymbol{R}_{A A^{\prime}}\right) \alpha_{A^{\prime} ; k l}(-2 k, k) V_{l j}\left(2 k, \boldsymbol{R}_{A^{\prime} B}\right) \mu_{B ; j}+\left[A \leftrightarrow A^{\prime}\right] .
\end{gathered}
$$

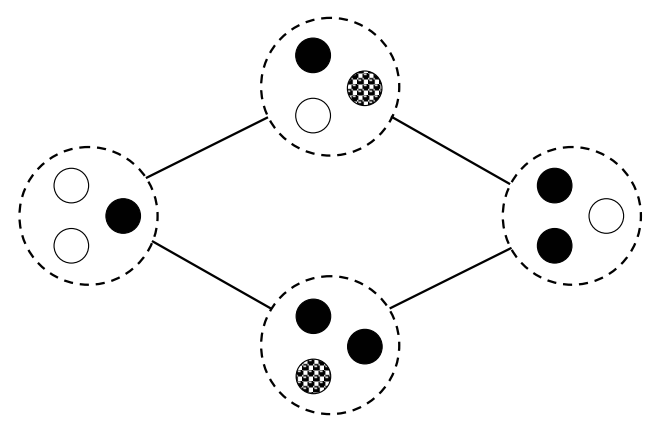

Fig. 3. Two fundamental mechanisms for energy pooling, from an initial system state on the left, through representative intermediate states, to the final state on the right. In each encircled depiction, closed circles on the left denote donors $(A$ and $A$ ), on the right an acceptor $B$. Black infill indicates a ground state, white a populated excited state, and patterning a virtual state. The upper route signifies cooperative pooling, the lower one accretive; the exhibited intermediate states are representative of a large set. In contrast to Fig. 2, in this collapsed and simplified statesequence diagram, each connector accommodates both virtual photon creation and annihilation. 


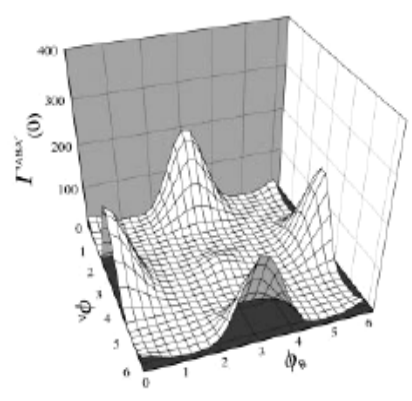

(a)

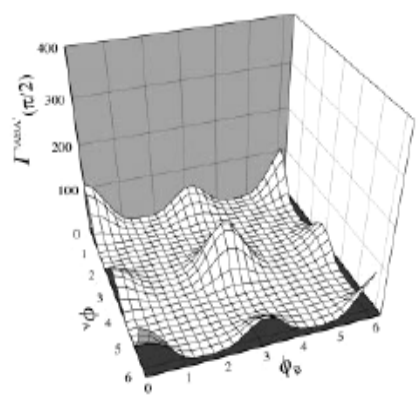

(b)

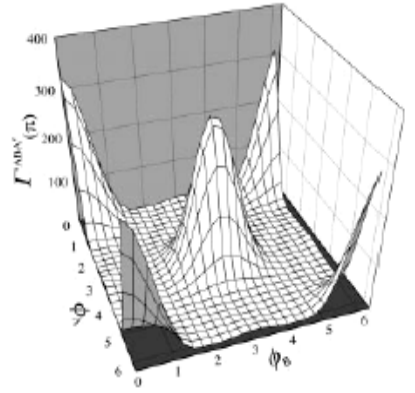

(c)

Fig. 4. Variation in efficiency of cooperative energy transfer in a twin-donor system with collinear geometry, as a function of the angles made by the transition moments of the two (outer) donors, $\varphi_{A}=\varphi_{A}$, and that of the (central) acceptor, $\varphi_{B}$, relative to the system axis. The angle $\theta$ between the plane containing the axis and the donor moment, and another containing the axis and the acceptor moment, takes the values: $(a) 0 ;(b) \pi / 2 ;(c) \pi[60]$.

In the cooperative mechanism, single-photon transitions characterize each donor transition, and the excitation of the acceptor follows two-photon selection rules - as is reflected in the two-photon absorption tensor $\sigma$ in (18). Accretive pooling requires the donor decay to satisfy both single- and two-photon selection rules. By way of illustration Fig. 4 shows, for a twin-donor system having a collinear geometry, $A^{\prime} B A$, plots of the variation in efficiency of cooperative energy transfer as a function of the chromophore orientations. In such a system the distance between the outer donors essentially precludes the accretive mechanism; in other cases both cooperative and accretive transfer need to be accommodated in the calculations.

The physical description of such complex processes is technically difficult; it needs to be emphasized that the rate of energy harvesting in multichromophore systems generally has contributions not only associated with each accretive or cooperative route individually, nor simply their combinations; other important terms result from quantum interference. The latter effect may of course produce either an enhancement or a reduction in the overall energy harvesting efficiency. The conceptualization of multichromophore energy pooling can be considerably simplified through the application of interaction-pair terminology [61]. The formalism is not only calculationally expedient, it can obviate a number of difficult semantic problems in the correct description of multichromophore interactions. Only detailed QED calculations properly reveal the extent to which the different quantum channels - such as the cooperative and accretive routes that connect the same initial and final states - are interlaced.

\section{DENDRIMERIC MATERIALS}

Dendrimers are repeatedly branched nanoscale polymers, also known as nanostars or functional cascade molecules [62-66], whose quasi-fractal geometry and large number of chemically similar chromophores built around a core approaches an ideal type of structure for energy harvesting, expediting highly efficient, ultrafast energy transport [66, 67]. The core is often a lanthanide ion, for reasons that will be expanded upon in the next Section, but other transition metal ions can also be employed [68]. The typical example shown in Fig. 5 should be viewed with a caveat: such commonplace planar depictions misrepresent the threedimensional folding that increasingly takes place with successive generations. In any such dendrimer the synthetic route generally builds outwards through successive generations, each further expanded to form a new outer shell by peripheral functionalization with additional 
groups. The resulting proliferation of chromophores on the outermost shell assists the photon capture efficiency of the dendrimer, though the associated generation-dependence in the distribution of inter-chromophore distances can reduce the efficiency of energy transfer [69]. In ideal cases the requisite spectroscopic gradient directed towards the core is established through the generation-dependent electronic environment of chemically similar chromophores [70-73]; most work on dendrimers has utilized branching motifs of vertex degree 3 and 4, based on tri-substituted benzene [63] and porphyrin rings [74], respectively. A recent development has been the construction of dendrimers whose internal cavities host smaller dye molecules. Exploiting not only intramolecular but also intermolecular (dendrimer - dye) energy transfer, harvesting efficiencies estimated at around $80 \%$ have, for example, been reported for eosin embedded in a dendrimer with chromophore groups of four different types [75]. One major objective of current synthetic effort is to achieve, through funneling and trapping, the energy pooling that characterizes biological systems.

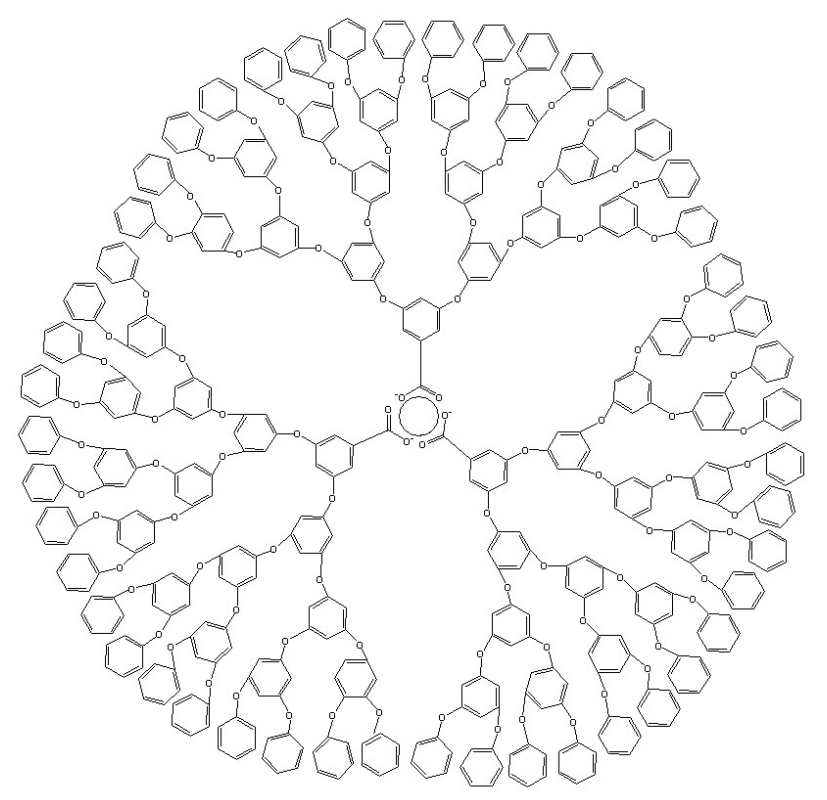

Fig. 5. Fifth-generation polyphenylether dendrimer, centred on a lanthanide ion such as $\mathrm{Er}^{3+}$ or $\mathrm{Tb}^{3+}$. Here the branching has a vertex degree (i.e. number of connectors per unit) equal to 3 . As a result of the flexibility in the ether linkages and the lone pair electrons on each oxygen, the dendrimer is in its natural state folded into quasi-spherical symmetry.

To model the multi-step flow of energy in dendrimers is a demanding challenge [76, 77], and a variety of calculational methods have been brought to bear on the problem. Often, simplifying assumptions are invoked - for example, in work by Blumen et al. [78], an exact solution has been derived for fractal polymers for which all chromophores have the same absorption cross-section, all rates of transfer between nearest neighbors also being considered equal. More radical approaches to the problem have also been attempted, such as modeling the diffusion of the excitation under a constant force as a continuum process [79], or using the Eyring (membrane permeation) model to treat energy flux as diffusion in a potential with thermal barriers [80].

A recently developed operator approach is founded upon an adjacency matrix representation, constructed on the basis of the chemical connectivity between individual 
chromophores [81-83]. A square matrix, whose order is equal to the number of chromophores, represents the propensities (probabilities associated with a specific time interval) for energy migration between the chromophores. This matrix operates upon a vector representation of the population conditions, each iteration representing an advance in time. Considerable simplification is effected, without compromising the fidelity of the model, by collapsing the representation into a reduced, shell basis whose order is generational number of the dendrimer. An advantage of the shell representation is that it obviates any false assumption of local symmetry suggested by chemical connectivity; this is a feature of particular relevance when issues of folding are entertained. For example, for a threegeneration dendrimer the RET propensity matrix in the shell basis, $\tilde{\mathbf{C}}$, can be expressed as follows:

$$
\tilde{\mathbf{C}}=\left(\begin{array}{cccc}
1-a_{3} & 2 \varepsilon_{3}{ }^{-1} a_{3} & 0 & 0 \\
a_{3} & 1-a_{2}-2 \varepsilon_{3}{ }^{-1} a_{3} & 2 \varepsilon_{2}^{-1} a_{2} & 0 \\
0 & a_{2} & 1-a_{1}-2 \varepsilon_{2}^{-1} a_{2} & 3 \varepsilon_{1}^{-1} a_{1} \\
0 & 0 & a_{1} & 1-3 \varepsilon_{1}^{-1} a_{1}-\xi
\end{array}\right)
$$

where $a_{i}$ is the propensity for energy transfer from a chromophore in the $i^{\text {th }}$ shell to another, to which it is chemically bonded, in the $(i-1)^{\text {th }}$ shell (or to the core, if $i=1$ ); $\varepsilon_{i}$ is the ratio of efficiencies for inward and outward energy transfer between the same pair of chromophores. The latter parameter is usually expressible through Eq. (13) as a simple function of the donor and acceptor chromophore line-shapes, and the corresponding pair spectroscopic gradients; often the simpler formulae (14) or (15) are applicable. This is where a shell-dependent variation, in the electronic influence exerted on each chromophore by its immediate neighbourhood, can play a decisive role, significantly increasing each $\varepsilon$ from its random-walk value of unity. Finally, $\xi$ quantifies losses associated with emission or irreversible energy utilisation at the core.

Results based on this model indicate a promising potential for representing the timedependence of energy flow towards the core, lending a new capacity to interpret experimentally determined kinetic data in terms of physically meaningful quantities with a clear molecular interpretation. For example, as indicated in Fig. 6(a), the striking effect of core decay (lower plots) indicates a time-window within which any acquired excitation must be irreversibly trapped. The significance of inter-shell directional transfer efficiency is also readily exhibited by results such as that shown in Fig. $6(b)$, where the increasingly strong dependence with successive generations of dendrimer growth is dramatically illustrated. In the more extensive results reported elsewhere, the effects of spectroscopic gradient, Stokes shift and three-dimensional folding are also detailed [83].

\section{RARE-EARTH DOPED SOLIDS}

One of the singular advantages afforded by a QED formulation of theory is that is provides a common ground for the representation of superficially very different processes. Moreover, it often reveals fundamental relationships between them. It may at first sight be surprising to discover in the field of solid-state crystal optics several of the mechanistic principles discussed in the foregoing description of dendrimeric materials, yet the links are extensive. The clearest case is frequency up-conversion, based on the same mechanisms of energy pooling that operate in multichromophore molecules [84]. In general, this fluorescence owes its origin to energy transfer mechanisms involving three chromophore/fluorophore sites, with two acting as donors and one as acceptor [85-87]. Exploiting such an effect, it is possible to tailor such materials specifically for stepwise or sequential laser frequency up-conversion. 

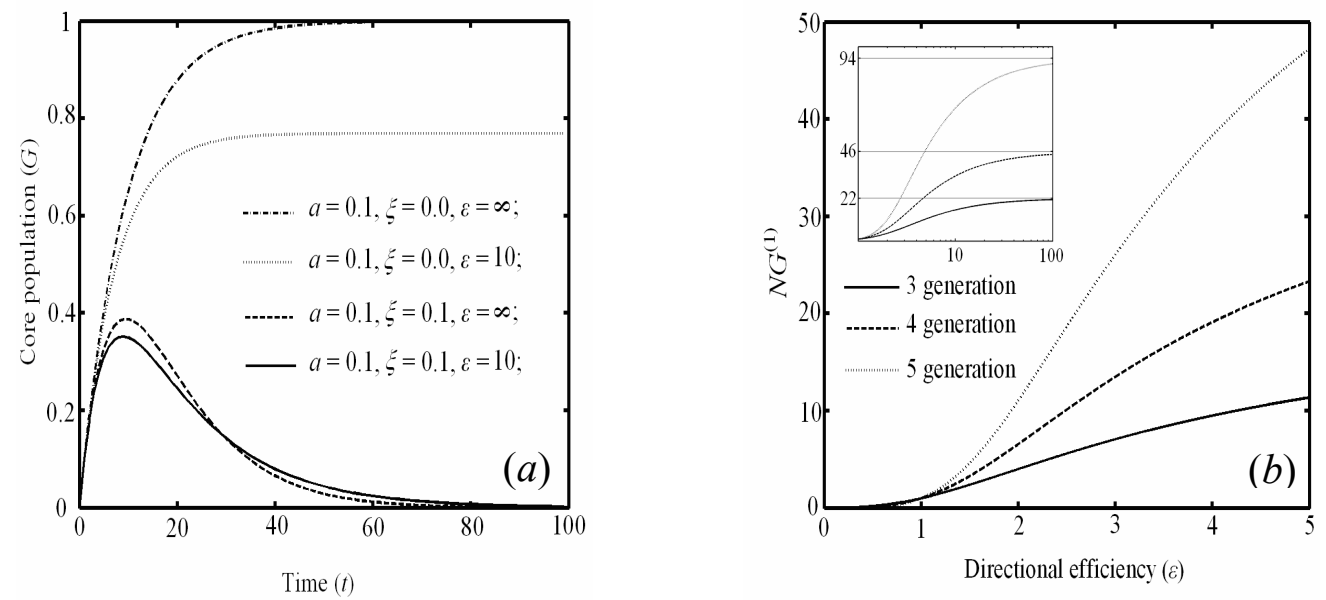

Fig. 6. (a) Extent of core excitation in a single generation dendrimer of vertex degree 3, plotted as a function of time, for different values of the parameters $a, \xi$ and $\varepsilon$, (b) Core excited state population (scaled by the number of chromophores) in dendrimers of vertex degree 3 , as a function of the inter-shell transfer efficiency, for 3-, 4- and 5-generation dendrimers.

Up-conversion is frequently manifest in observations of blue-shifted fluorescence; rareearth (lanthanide) doped crystals notably display the effect. The rare-earths afford propitious systems for experimental studies and applications [88] as they display strong $f-f$ (core $f$ electron) transitions associated with a redistribution of charge sufficiently close to the nucleus that the ionic environment exercises relatively little influence. As a consequence, their absorption and fluorescence spectra contain highly discrete, characteristic and well-resolved lines, with many falling in wavelength regions eminently suitable for laser excitation $[89,90]$. It should be emphasized that the materials under discussion here operate quite differently from conventional nonlinear optical crystals (where off-resonance input photons directly engage in pairwise or higher order coupling, not involving any optical excitation of the material). By contrast, energy pooling is non-parametric and generally involves losses; indeed, associated phonon generation can expedite transfer by modifying the selection rules.

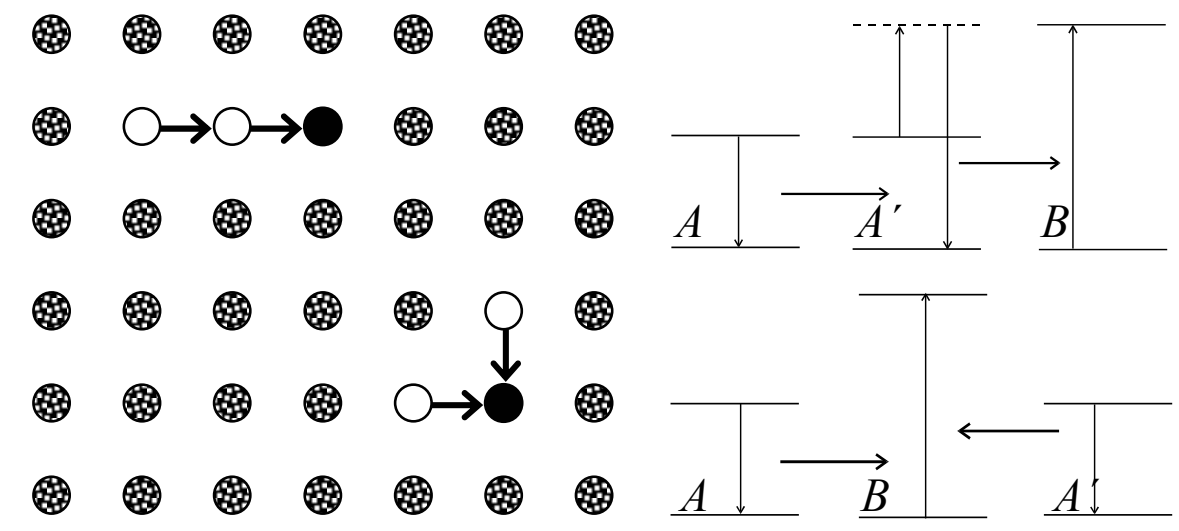

Fig. 7. Typical dopant geometry and energy level schemes for non-parametric up-conversion: accretive (above left, right); cooperative (below left, right). Here, the donor ions are identical. 
As with dendrimeric and other multi-chromophore pooling, both cooperative and accretive mechanisms may operate in a suitably doped crystal; inverse down-conversion processes may also arise [84, 91]. Equations (18) and (19) again apply, imposing their associated selection rules. If these rules allow, both mechanisms will contribute to the overall quantum amplitude for energy pooling. However, as illustrated in Fig.7, the spatial dispositions of the ions involved may dispose more towards one or the other mechanism - bearing in mind the inverse sixth power dependence of each energy transfer involved. Moreover the two donors need not be of the same chemical type.

Following optical excitation, the relative number densities of excited donors and acceptors is a significant determinant of mechanism, alongside the different optical selection rules that operate in each mechanism. For the most general case, consider a system based on three chemically different dopants $A, B$ and $C$ (also accommodating any case where the transitions involved differ, though there is an atomic equivalence). To effect energy pooling, $A$ and $B$ will play the role of donors and $C$ the acceptor. In determining the efficiency of energy pooling it is necessary to consider all permissible ion positions on the lattice, and also the number of ions $A, B$, and $C$, residing in their appropriate initial electronic states (excited states for the donors, ground state for the acceptor species), per unit cell. If these are written as $C_{X}$ for the component $X$, then the appropriate concentrations, for the case of a cubic crystal with cell length $a$, are expressible as $c_{X}=a^{-3} C_{X}$. Discounting any triad where two or three ions share identical lattice sites, and assuming random positioning of the salient ions, the lattice sums that feature in the ensemble rates yield the following energy harvesting efficiency factors [84]:

$$
\begin{aligned}
& \sum_{\text {lattice }} R_{A B}^{-6} R_{B C}^{-6}=\eta C_{A} C_{B}^{2} C_{C} a^{-12}=\eta c_{A} c_{B}^{2} c_{C}, \\
& \sum_{\text {lattice }} R_{B A}^{-6} R_{A C}^{-6}=\eta C_{A}^{2} C_{B} C_{C} a^{-12}=\eta c_{A}^{2} c_{B} c_{C}, \\
& \sum_{\text {lattice }} R_{A C}^{-6} R_{B C}^{-6}=\eta C_{A} C_{B} C_{C}^{2} a^{-12}=\eta c_{A} c_{B} c_{C}^{2} .
\end{aligned}
$$

which apply to the accretive process $A \rightarrow B \rightarrow C$, the accretive alternative $B \rightarrow A \rightarrow C$, and the cooperative case $A \rightarrow C \leftarrow B$, respectively. In the above results, $\eta$ is a structure-dependent numerical factor, here taking the value, $\eta=64.39$ (other results apply for lattices of different symmetry). The lattice weightings thus signify concentration factors for each of the participating ions in the necessary initial states. Where both accretive and cooperative pooling occur (which is possible when inversion symmetry is absent), quantum interference terms have also to be accommodated in the rate equations for energy harvesting. For ions randomly disposed on a cubic lattice, contributions to the rate from such interferences are just as important as the fully accretive or fully cooperative kind. In connection with conventional (single-donor) energy transfer, calculations based on this method have been applied extensively to crystal systems $[92,93]$, with some work also accounting for higher multipole couplings and the exchange interaction $[94,95]$. Similar statistically based calculations have also been performed for amorphous solids [96]. Examples of twin-donor energy pooling in up-conversion are rife, and not necessarily limited to systems containing more than one species of rare-earth ion. The occurrence of such a process was, for example, first identified in systems involving a concerted action amongst three electronically excited $\operatorname{Pr}^{3+}$ ions, in 
which two ions decay and the third is further excited through the acquisition of their combined energy [97,98]. An iso-ionic process also accounts for green and red emissions from $\mathrm{Er}^{3+}$ in fluorite-type crystals [99]. Although it is beyond the scope of the present review, there is also an extensive literature reporting energy pooling at high intensities, associated with multiphoton absorption in individual donors - see refs [100-103] and citations therein; a recent study of the three-photon excited emission of green light from a $\mathrm{Tb}^{3+}$-organic complex is a fine example [104].

\section{CONCLUSION}

This review has focused on key elements of the theory underlying optical energy harvesting, addressing the primary processes that direct, convey and pool excitation energy initially acquired on photon absorption. In a variety of aspects there is a remarkable and highly significant interplay between the structures and fundamental mechanisms operating in energy harvesting systems. The relative efficiency of the mechanisms themselves depend on major structural parameters - in particular the strong dependences on the relative orientations of chromophores and on inter-chromophore distances, as well as on electrical properties such as static and transition dipole moments, and polarizabilities. Although the search for more efficient means of harvesting solar power has driven much of the activity in this field, the principles that are emerging from the theory have applications that extend well beyond implementation in the design of new materials for this purpose alone.

The whole science of photophysics in nanostructured molecular and dielectric materials is undergoing a transformation through the emergence of highly detailed representations of energy transfer mechanisms, many of which were scarcely known ten years ago. Developing the theory based on quantum electrodynamics proves to have several virtues, beyond intrinsic rigor. QED provides a common basis for casting the different mechanistic features into quantitative form; it allows parallels to be identified between processes occurring in physically very different types of medium, such as natural photosynthetic apparatus, dendrimeric polymers and lanthanide-doped crystals; it also affords fresh insights into the basic mechanisms. In its detail, there is plenty of scope to further explore the linkage between structure and mechanism. For example, it is a striking facet of the adjacency matrix representation of energy flow in dendrimers that its matrix basis, determined by principles of chemical connectivity between groups, is isomorphous with the corresponding collapsed state-sequence diagram for energy pooling. This is just one of several illuminating and potentially simplifying features whose exploitation is the subject of ongoing study. As the science progresses, a better comprehension is the first-fruit of every successive intellectual harvest.

\section{Acknowledgments}

I am grateful to David Bradshaw for many helpful comments. Research in the QED group at UEA is funded by the Engineering and Physical Sciences Research Council, and the Leverhulme Trust.

\section{References}

[1] S. F. Baldwin, "Renewable energy: progress and prospects," Phys. Today 55(4), 62-67 (2002) [doi:10.1063/1.1480784].

[2] G. W. Crabtree and N. S. Lewis, "Solar energy conversion," Phys. Today 60(3), 37-42 (2007) [doi:10.1063/1.2718755].

[3] M. A. Green, Third Generation Photovoltaics: Advanced Solar Energy Conversion, Springer, Berlin (2003). 
[4] S. Guenes and N. S. Saricifci, "Hybrid solar cells," Inorg. Chimica Acta 361, 581-588 (2008) [doi:10.1016/j.ica.2007.06.042].

[5] V. S. Arunachalam and E. L. Fleischer, "The global energy landscape and materials innovation," Mater. Res. Soc. Bull. 33, 264-276 (2008).

[6] J. Barber, "Biological solar energy," Phil. Trans. R. Soc. Lond. A 365, 1007-1023 (2007) [doi:10.1098/rsta.2006.1962].

[7] B. Grimm, R. J. Porra, W. Rüdiger, and H. Scheer, Chlorophylls and Bacteriochlorophylls, Springer, Dordrecht, The Netherlands (2006) [doi: 10.1007/14020-4516-6].

[8] D. L. Andrews, Energy Harvesting Materials, World Scientific, River Edge, NJ, USA (2005).

[9] L. Mateu and F. Moll, "Review of energy harvesting techniques and applications for microelectronics," Proc. SPIE 5837, 359-373 (2005) [doi:10.1117/12.613046].

[10] T. A. Anderson and D. W. Sexton, "A vibration energy harvesting sensor platform for increased industrial efficiency," Proc. SPIE 6174, 61741Y (2006) [doi:10.1117/12.659586].

[11] Y. Zhang, L. Li, H. San, and X. Chen, "Piezoelectric capacitive power generator from vibration energy," Proc. SPIE 6836, 68360Y (2007). [doi:10.1117/12.756131].

[12] C. Mo, L. J. Radziemski, and W. W. Clark, "Performance comparison of implantable piezoelectric energy harvesters," Proc. SPIE 6928, 69280C (2008) [doi:10.1117/12.776293].

[13] M. Zhang, D. Brignac, P. Ajmera, and K. Lian, "A low-frequency vibration-toelectrical energy harvester," Proc. SPIE 6931, 69310S (2008) [doi:10.1117/12.775920V].

[14] G. D. Scholes and K. P. Ghiggino, "Mechanisms of excitation transfer in multichromophoric systems," J. Photochem. Photobiol. A - Chem. 80, 355-362 (1994) [doi:10.1016/1010-6030(93)01036-2].

[15] G. D. Scholes and K. P. Ghiggino, "Rate expressions for excitation transfer. 4. Energy migration and superexchange phenomena," $J$ Chem. Phys. 103, 8873-8883 (1995). [doi: 10.1063/1.470076].

[16] E. K. L. Yeow, D. J. Haines, K. P. Ghiggino, and M. N. Padden-Row, "Electronic energy transfer in multichromophoric arrays. A sequential and superexchange dynamics study," J. Phys. Chem. A 103, 6517-6524 (1999) [doi:10.1021/jp991113b].

[17] E. K. L. Yeow and K. P. Ghiggino, "Electronic energy transfer in multichromophoric arrays. The effects of disorder on superexchange coupling and energy transfer rate," $J$. Phys. Chem. A 104, 5825-5836 (2000) [doi:10.1021/jp994218n].

[18] G. D. Scholes, "Long-range resonance energy transfer in molecular systems," Annu. Rev. Phys. Chem. 54, 57-87(2003) [doi: 10.1146/annurev.physchem.54.011002.103746].

[19] W. J. D. Beenken and T. Pullerits, "Spectroscopic units in conjugated polymers: a quantum chemically founded concept?" J. Phys. Chem. B 108, 6164-6169 (2004) [doi:10.1021/jp0373321].

[20] C. Bacchiocchi, E. Hennebicq, S. Orlandi, L. Muccioli, D. Beljonne, and C. Zannoni, "'Reduced" distributed monopole model for the efficient prediction of energy transfer in condensed phases," J. Phys. Chem. B 112, 1752-1760 (2008) [doi:10.1021/jp076732w].

[21] D. L. Dexter, "A theory of sensitized luminescence in solids," J. Chem. Phys. 21, 836850 (1953) [doi:10.1063/1/1699044].

[22] T. Förster, "Zwischenmolekulare Energiewanderung und Fluoreszenz," Annalen Phys. 2, 55-75 (1948) [doi:10.1002/andp.19484370105]. 
[23] S. A. Latt, H. T. Cheung, and E. R. Blout, "Energy transfer - a system with relatively fixed donor-acceptor separation," J. Amer. Chem. Soc. 87, 995-1003 (1965) [doi:10.1021/ja01083a011].

[24] A. A. Demidov and D. L. Andrews, Encyclopedia of Chemical Physics and Physical Chemistry, Vol. 3, pp. 2701-2715, J. H. Moore and N. D. Spencer, Eds., Institute of Physics, Bristol, UK (2001) [doi:10.1887/0750303131/b984v3c27].

[25] D. L. Andrews and J. Rodríguez, "Resonance energy transfer: spectral overlap, efficiency and direction," J. Chem. Phys. 127, 084509 (2007) [doi:10.1063/1.2759489].

[26] C. Galli, K. Wynne, S. M. Lecours, M. J. Therien, and R. M. Hochstrasser, "Direct measurement of electronic dephasing using anisotropy," Chem. Phys. Lett. 206, 493499 (1993) [doi:10.1016/0009-2614(93)80174-N].

[27] B. W. van der Meer, in Resonance Energy Transfer, pp. 151-172, D. L. Andrews and A. A. Demidov, Eds., Wiley, New York (1999).

[28] D. P. Craig and T. Thirunamachandran, Molecular Quantum Electrodynamics, Dover, New York (1998).

[29] P. W. Milonni, The Quantum Vacuum, Academic Press, Boston (1994).

[30] R. G. Woolley, "Gauge invariance in non-relativistic electrodynamics," Proc. R. Soc. London, Ser A 456, 1803-1819 (2000) [doi:10.1098/rspa.2000.0587].

[31] J. S. Avery, "Resonance energy transfer and spontaneous photon emission," Proc. Phys. Soc. 88, 1-8 (1966) [doi:10.1088/0370-1328/88/1/302].

[32] L. Gomberoff and E. A. Power, "The resonance transfer of excitation," Proc. Phys. Soc. 88, 281-284 (1966) [doi:10.1088/0370-1328/88/2/302].

[33] D. L. Andrews and B. S. Sherborne, "Resonant excitation transfer: a quantum electrodynamical study," J. Chem. Phys. 86, $4011-4017$ (1987) [doi:10.1063/1.451910].

[34] D. L. Andrews, "A unified theory of radiative and radiationless molecular energy transfer," Chem. Phys. 135, 195-201 (1989) [doi:10.1016/0301-0104(89)87019-3].

[35] G. Juzeliūnas and D. L. Andrews, "Quantum electrodynamics of resonance energy transfer," Adv. Chem. Phys. 112, 357-410 (2000) [doi:10.1002/978047141717.ch4].

[36] G. J. Daniels, R. D. Jenkins, D. S. Bradshaw and D. L. Andrews, "Resonance energy transfer: the unified theory revisited," J. Chem. Phys. 119, 2264-2274 (2003) [doi:10.1063/1/1579677].

[37] M. Babiker, E. A. Power, and T. Thirunamachandran, "Generalization of PowerZienau-Woolley transformation in quantum electrodynamics and atomic field equations," Proc. R. Soc. A 338, 235-249 (1974) [doi:10.1098/rspa.1974.0084].

[38] W. Demtröder, Atoms, Molecules and Photons, pp. 108-109, Springer, Berlin (2006).

[39] A. Salam, "A general formula for the rate of resonant transfer of energy between two electric multipole moments of arbitrary order using molecular quantum electrodynamics," J. Chem. Phys. 122, 044112 (2005) [doi:10.1063/1.1830430].

[40] G. D. Scholes and D. L. Andrews, "Damping and higher multipole effects in the quantum electrodynamical model for electronic energy transfer in the condensed phase," J. Chem. Phys. 107, 5374-5384 (1997) [doi:10.1063/1.475145].

[41] D. L. Andrews, "Mechanistic principles and applications of resonance energy transfer," Canad. J. Chem. 86, 855-870 (2008) [doi: 10.1139/V08-020].

[42] D. Brühwiler, L.-Q. Dieu, and G. Calzaferri, "Nanochannel materials for quantum solar energy conversion devices," Chimia 61, 820-822 (2007) [doi:10.2533/chimia.2007.820].

[43] A. Ajayaghosh, V. K. Praveen and C. Vijayakumar, "Organogels as scaffolds for excitation energy transfer and light harvesting," Chem. Soc. Rev. 37, 109-122 (2008) [doi:10.1039/b704456a].

[44] I. Akai, A. Okada, K. Kanemoto, T. Karasawa, H. Hashimoto, and M. Kimura, "Quenching of energy transfer by freezing molecular vibrations in light-harvesting 
small dendrimer," J. $\quad$ Lumin. 119-120, 283-287 [doi:10.1016/j.jlumin.2006.01.014].

[45] C.-P. Hsu, G. R. Fleming, M. Head-Gordon, and T. Head-Gordon, "Excitation energy transfer in condensed media," J. Chem. Phys. 114, 3065-3072 (2001) [doi:10.1063/1.1338531].

[46] K. P. Ghiggino, E. K. L. Yeow, D. J. Haines, G. D. Scholes, and T. A. Smith, "Mechanisms of excitation energy transport in macromolecules," J. Photochem. Photobiol. A: Chem. 102, 81-86 (1996) [doi:10.1016/S1010-6030(96)04374-2].

[47] E. Hindin, R. A. Forties, R. S. Loewe, A. Ambroise, C. Kirmaier, D. F. Bocian, J. S. Lindsey, D. Holten and R. S. Knox, "Excited-state energy flow in covalently linked multiporphyrin arrays: the essential contribution of energy transfer between nonadjacent chromophores," J. Phys. Chem. B 108, 12821-12832 (2004) [doi:10.1021/jp047803j].

[48] G. J. Daniels and D. L. Andrews, "The electronic influence of a third body on resonance energy transfer," J. Chem. Phys. 116, 6701-6712 (2002) [doi:10.1063/1.1461819].

[49] B. Fückel, A. Köhn, M. E. Harding, G. Diezemann, G. Hinze, T. Basché, and J. Gauss, Theoretical investigation of electronic excitation energy transfer in bichromophoric assemblies," J. Chem. Phys. 128, 074505 (2008) [doi:10.1063/1.2829531].

[50] L. D. Bakalis and J. Knoester, "Linear absorption as a tool to measure the exciton delocalization length in molecular assemblies," J Lumin. 87-89, 66-70 (2000) [doi:10.1016/S0022-2313(99)00231-8].

[51] G. D. Scholes and G. R. Fleming, "On the mechanism of light harvesting in photosynthetic purple bacteria: B800 to B850 energy transfer," J. Phys. Chem. B 104, 1854-1868 (2000) [doi:10.1021/jp9934351].

[52] S. Gnanakaran, R. Haran, R. Kumble, and R. M. Hochstrasser, "Energy transfer and localization: applications to photosynthetic systems," in Resonance Energy Transfer, pp. 308-365, D. L. Andrews and A. A. Demidov, Eds., Wiley, New York (1999).

[53] S. Savikhin, D. R. Buck, and W. S. Struve, "The Fenna-Matthews-Olsen protein: a strongly coupled photosynthetic antenna," in Resonance Energy Transfer, pp. 399-434, D. L. Andrews and A. A. Demidov, Eds., Wiley, New York (1999).

[54] H. van Amerongen, L. Valkunas, and R. van Grondelle, Photosynthetic Excitons, World Scientific, New Jersey (2000).

[55] D. Kuciauskas, P. A. Liddell, S. Lin, T. E. Johnson, S. J. Weghorn, J. S. Lindsey, A. L. Moore, T. A. Moore, and D. Gust, "An artificial photosynthetic antenna-reaction center complex," J. Amer. Chem. Soc. 121, 8604-8614 (1999) [doi:10.1021/ja991255j].

[56] G. D. Scholes, X. J. Jordanides, and G. R. Fleming, "Adapting the Förster theory of energy transfer for modeling dynamics in aggregated molecular assemblies," J. Phys. Chem. B 105, 1640-1651 (2001) [doi:10.1021/jp003571m].

[57] V. A. Morozov, "Redistribution of photoexcitation energy between chromophores in a complex molecule and the "light-collecting antenna" effect," Russ. J. Phys. Chem. 75, 246-254 (2001).

[58] R. D. Jenkins and D. L. Andrews, "Three-center systems for energy pooling: quantum electrodynamical theory," J. Phys. Chem. A 102, 10834-10842 (1998) [doi:10.1021/jp983071h].

[59] C. A. Sierra and P. M. Lahti, "A simple multichromophore design for energy transfer in distyrylbenzenes with pyrene pendants," J. Phys. Chem. A 110, 12081-12088 (2006) [doi:10.1021/jp060543g].

[60] R. D. Jenkins and D. L. Andrews, "Orientation factors in three-centre energy pooling," Phys. Chem. Chem. Phys. 2, 2837-2843 (2000) [doi:10.1039/b002288h]. 
[61] R. D. Jenkins and D. L. Andrews, "Four-center energy transfer and interaction pairs: molecular quantum electrodynamics," J. Chem. Phys. 116, 6713-6724 (2002) [doi:10.1063/1/1461820].

[62] A. Adronov and J. M. J. Fréchet, "Light-harvesting dendrimers," Chem. Commun., 1701-1710 (2000) [doi:10.1039/b005993p].

[63] A. Archut and G. Vögtle, "Functional cascade molecules," Chem. Soc. Rev. 27, 233240 (1998) [doi:10.1039/a827233z].

[64] T. Minami, S. Tretiak, V. Chernyak, and S. Mukamel, "Frenkel-exciton Hamiltonian for dendrimeric nanostar," $J$ Lumin. 87-89, 115-118 (2000) [doi:10.1016/S00222313(99)00242-2].

[65] V. Balzani, P. Ceroni, M. Maestri, and V. Vincinelli, "Light-harvesting dendrimers," Curr. Opinion. Chem. Biol. 7, 657-665 (2003) [doi:10.1016/j.cbpa.2003.10.001].

[66] M. I. Ranasinghe, O. P. Varnavski, J. Pawlas, S. I. Hauck, J. Louie, J. F. Hartwig, and T. Goodson III, "Femtosecond excitation energy transport in triarylamine dendrimers," J. Am. Chem. Soc. 124, 6520-6521 (2002) [doi:10.1021/ja025505z].

[67] T. Goodson III, "Optical excitations in organic dendrimers investigated by timeresolved and nonlinear optical spectroscopy," Acc. Chem. Res. 38, 99-107 (2005) [doi: 10.1021/ar020247w].

[68] J. Larsen, F. Puntoriero, T. Pascher, N. McClenaghan, S. Campagna, E. Åkesson, and V. Sundström, "Extending the light-harvesting properties of transition-metal dendrimers," ChemPhysChem 8, 2643-2651 (2007) [doi:10.1002/cphc.200700539].

[69] M. Lor, R. De, S. Jordens, G. De Belder, G. Schweitzer, M. Cotlet, J. Hofkens, T. Weil, A. Herrmann, K. Müllen, M. Van Der Auweraer, and F. C. De Schryver, "Generation-dependent energy dissipation in rigid dendrimers studied by femtosecond to nanosecond time-resolved fluorescence spectroscopy," J. Phys. Chem. A 106, 20832090 (2002) [doi:10.1021/jp012310p].

[70] M. R. Shortreed, S. F. Swallen, Z. Y. Shi, W. H. Tan, Z. F. Xu, C. Devadoss, J. S. Moore, and R. Kopelman, "Directed energy transfer funnels in dendrimeric antenna supermolecules," J. Phys. Chem. B 101, 6318-6322 (1997) [doi:10.1021/jp9705986].

[71] A. Bar-Haim and J. Klafter, "Dendrimers as light harvesting antennae," J. Lumin. 7677, 197-200 (1998) [doi:10.1016/S0022-2313(97)00150-6].

[72] A. Bar-Haim and J. Klafter, "Geometric versus energetic competition in light harvesting by dendrimers," $J$. Phys. Chem. B 102, 1662-1664 (1998) [doi:10.1021/jp980174r].

[73] S. F. Swallen, Z.-Y. Shi, W. Tan, Z. Xu, J. S. Moore, and R. Kopelman, "Exciton localisation hierarchy and directed energy transfer in conjugated linear aromatic chains and dendrimeric supermolecules," J. Lumin. 76-77, 193-196 (1998) [doi:10.1016/S0022-2313(97)00149-X].

[74] P. G. van Patten, A. P. Shreve, J. S. Lindsey, and R. J. Donohoe, "Energy-transfer modeling for the rational design of multiporphyrin light-harvesting arrays," $J$. Phys. Chem. B 102, 4209-4216 (1998) [doi:10.1021/jp972304m].

[75] U. Hahn, M. Gorka, F. Vögtle, V. Vicinelle, P. Ceroni, M. Maestri, and V. Balzani, "Light-harvesting dendrimers: efficient intra- and intermolecular energy-transfer processes in a species containing 65 chromophoric groups of four different types," Angew. Chem. Int. Ed. 41, 3595-3598 (2002) [doi:10.1002/15213773(20021004)41:19<3595::AID-ANIE3595>3.0.CO;2-B].

[76] M. Nakano, M. Takahata, H. Fujita, S. Kiribayashi, and K. Yamaguchi, "Exciton migration dynamics in a dendritic molecular aggregate," Chem. Phys. Lett. 323, 249256 (2000) [doi:10.1016/S0009-2614(00)00506-6].

[77] J. L. Bentz and J.J. Kozak, "Influence of geometry on light harvesting in dendrimeric systems. II. $\mathrm{n}^{\text {th }}$-nearest neighbor effects and the onset of percolation," J. Lumin. 121, 62-74 (2006) [doi:10.1016/j.jlumin.2005.10.004]. 
[78] A. Blumen, A. Volta, A. Jurjiu, and Th. Koslowski, "Energy transfer and trapping in regular hyperbranched macromolecules," Physica A. 356, 12-18 (2005) [doi:10.1016/j.physa.2005.05.005].

[79] S. M. Vlaming, D. J. Heijs, and J. Knoester, "Transport of optical excitations on dendrimers in the continuum approximation," J. Lumin. 111, 349-358 (2005) [doi:10.1016/j/jlumin.2004.10.015].

[80] D. Rana and G. Gangopadhyay, "Studies on energy transfer in dendrimer supermolecule using classical random walk model and Eyring model," J. Chem. Phys. 118 434-443 (2003) [doi:10.1063/1/1526095].

[81] D. L. Andrews and S. Li, "Adjacency matrix formulation of energy flow in dendrimeric polymers," Proc. SPIE 6328, 63280V (2006) [doi:10.1117/12.678038].

[82] D. L. Andrews and S. Li, "Energy flow in dendrimers: An adjacency matrix representation," Chem. Phys. Letts. 433, 239-243 (2006) [doi:10.1016/j.cplett.2006.11.049].

[83] D. L. Andrews, S. Li, J. Rodríguez and J. Slota, "Development of the energy flow in light-harvesting dendrimers," J. Chem. Phys. 127, 134902 (2007) [doi:10.1063/1.2785175].

[84] D. L. Andrews and R. D. Jenkins, "A quantum electrodynamical theory of three-center energy transfer for upconversion and downconversion in rare earth doped materials," $J$. Chem. Phys. 114, 1089-1100 (2001) [doi:10.1063/1.1323958].

[85] F. Auzel, "Upconversion processes in coupled ionic systems," J. Lumin. 45, 341-345 (1990) [doi:10.1016/0022-2313(90)90189-I].

[86] M. Chua and P. A. Tanner, "Three-body energy transfer processes of lanthanide ions in crystals," J. Lumin. 66-67, 203-207 (1995) [doi:10.1016/0022-2313(95)00137-9].

[87] X. Zhang, J.-P. Jouart, and G. Mary, "Energy transfer upconversion in $\mathrm{Ho}^{3+}$ and $\mathrm{Ho}^{3+}$, $\mathrm{Yb}^{3+}$ doped $\mathrm{CdF}_{2}$ crystals," J. Phys. C: Condens. Matter 10, $493-500$ (1998) [doi:10.1088/0953-8984/10/027].

[88] A. Meijerink, R. Wegh, P. Vergeer, and T. Vlugt, "Photon management with lanthanides," Opt. Mater. 28, 575-581 (2006) [doi:10.1016/j.optmat.2005.09.055].

[89] B. G. Wybourne, Spectroscopic Properties of Rare Earths, Wiley Interscience, New York (1965).

[90] V. V. Ovsyankin, Spectroscopy of Solids Containing Rare Earth Ions, A. A. Kaplyanskii and R. M. Macfarlane, Eds., Elsevier, Amsterdam (1987).

[91] P. Vergeer, T. J. H. Vlugt, M. H. F. Kox, M. I. Den Hertog, J. P. J. M. Van Der Herden, and A. Meijerink, "Quantum cutting by cooperative energy transfer in $\begin{array}{lllllll}\mathrm{Yb}_{\mathrm{x}} \mathrm{Y}_{1-\mathrm{x}} \mathrm{PO}_{4}: \mathrm{Tb}^{3+}, " & \text { Phys. Rev. } & B & \text { 71, } & 014119 & \text { (2005) }\end{array}$ [doi:10.1103/PhysRevB.71.014119].

[92] S. O. Vasquez, "Energy transfer processes in organized media. I. a crystal model for cubic sites," J. Chem. Phys. 104, $7652-7657$ (1996) [doi:10.1063/1.471480].

[93] S. O. Vasquez, "Energy transfer processes in organized media. II. generalization of the crystal model for dipole-dipole interactions in cubic sites," J. Chem. Phys. 106, 86648671 (1997) [doi:10.1063/1.473928].

[94] S. O. Vasquez, "Crystal model for energy-transfer processes in organized media: higher-order electric multipolar interactions," Phys. Rev. B 60, 8575-8585 (1999) [doi:10.1103/PhysRevB.60.8575].

[95] C. Z. Hadad and S. O. Vasquez, "Energy-transfer processes induced by exchange interactions," Phys. Rev. B 60, 8586-8594 (1999) [doi:10.1103/PhysRevB.60.8586].

[96] C. Z. Hadad and S.O. Vásquez, "Statistical approach to the transient up-converted population in monodoped amorphous solids," Phys. Chem. Chem. Phys. 5, 3027-3033 (2003) [doi:10.1039/b302964f].

[97] L.-S. Lee, S. C. Rand, and A. L. Schawlow, "Cooperative energy-transfer among $\operatorname{Pr}^{3+}$ ions in $\mathrm{LaF}_{3}$," Phys. Rev. B 29, 6901-6906 (1984) [doi:10.1103/PhysRevB.29.6901]. 
[98] A. Lezama, M. Oriá, J. R. Rios Leite, and C. B. De Araújo, "Triad spectroscopy via ultraviolet upconversion in $\mathrm{Pr}^{3+}-\mathrm{LaF}_{3}$, Phys. Rev. B 32, $7139-7142$ (1985) [doi:10.1103/PhysRevB.32.7139].

[99] J. P. Jouart and G. Mary, "Upconversion in $\mathrm{Er}^{3+}$-doped fluorite-type crystals pumped by $1.5 \mu \mathrm{m}$ tunable diode-laser," J. Lumin. 46, 39-45 (1990) [doi:10.1016/00222313(90)90080-U].

[100] P. Allcock and D. L. Andrews, "Two-photon fluorescence: resonance energy transfer," J. Chem. Phys. 108, 3089-3095 (1998) [doi:10.1063/1.475706].

[101] D. L. Andrews and R. D. Jenkins, "Nonlinearities in energy harvesting media," Proc. SPIE 4467, 297-306 (2001) [doi:10.1117/12.432943].

[102] D. L. Andrews and D. S. Bradshaw, "Optically nonlinear energy transfer in lightharvesting dendrimers," J. Chem. Phys. 121, 2445-2454 (2004) [doi:10.1063/1.1769354].

[103] D. L. Bradshaw and D. L. Andrews, "Competing mechanisms for energy transfer in two-photon absorbing systems," Chem. Phys. Letts 430, 191-194 (2006) [doi:10.1016/j.cplett.2006.08.116].

[104] G.-L. Law, W.-M. Kwok, W.-T. Wong, K.-L. Wong, and P. A. Tanner, "Terbium luminescence sensitized through three-photon excitation in a self-assembled unlinked antenna," J. Phys. Chem. B 111, 10858-10861 (2007) [doi:10.1021/jp0755185]. 